\title{
A Fast On-Line Neural-Network Training Algorithm for a Rectifier Regulator
}

\author{
Farrukh Kamran, Student Member, IEEE, Ronald G. Harley, Fellow, IEEE, Bruce Burton, Student Member, IEEE, \\ Thomas G. Habetler, Senior Member, IEEE, and Martin A. Brooke, Member, IEEE
}

\begin{abstract}
This paper addresses the problem of deadbeat control in fully controlled high-power-factor rectifiers. Improved deadbeat control can be achieved through the use of neuralnetwork-based predictors for the input-current reference to the rectifier. In this application, on-line training is absolutely required. In order to achieve sufficiently fast on-line training, a new random-search algorithm is presented and evaluated. Simulation results show that this type of network training yields equivalent performance to standard backpropagation training. Unlike backpropagation, however, the random weight change (RWC) method can be implemented in mixed digital/analog hardware for this application. The paper proposes a very large-scale integration (VLSI) implementation which achieves a training epoch as low as $8 \mu \mathrm{s}$.
\end{abstract}

Index Terms - Current regulator, neural network, on-line training, power electronic rectifier.

\section{INTRODUCTION}

$\mathbf{R}$ ECTIFIERS are an integral part of almost every power electronic system that connects to the utility supplying ac. In the industrial power range, mainly three-phase rectifiers are employed with either one-way or bidirectional power flow capabilities. In the case of the three-phase bidirectional rectifiers, the boost-derived six-switch topology has been adopted by most researchers because of its advantages. The most popular method of control for these rectifiers consists of two nested control loops-a slower outer loop that computes reference signals for the ac-input currents and a faster inner current regulator that forces the actual ac-input currents to follow these desired trajectories as illustrated in Fig. 1. This control method guarantees stability and also achieves high control bandwidth and good steady-state performance. The input-current reference $I_{\mathrm{dq}}^{\mathrm{ref}}$ is derived so as to balance the instantaneous ac-input and dc-output powers and to keep the input currents proportional to the corresponding input phase voltages. The input power demand is computed as a combination of the instantaneous output power and the dc-bus voltage error.

Manuscript received May 15, 1996; revised January 6, 1997. Recommended by Associate Editor, A. Kawamura.

F. Kamran is with the Faculty of Electronics, Ghulam Ishaq Khan Institute of Engineering Sciences and Technology, Pakistan.

T. G. Habetler and M. A. Brooke are with the Georgia Institute of Technology, School of Electrical and Computer Engineering, Atlanta, GA 30332 USA (e-mail: thabetler@ee.gatech.edu).

R. G. Harley and B. Burton are with the Department of Electrical Engineering, University of Natal, Durban, 4001 South Africa.

Publisher Item Identifier S 0885-8993(98)01941-3.

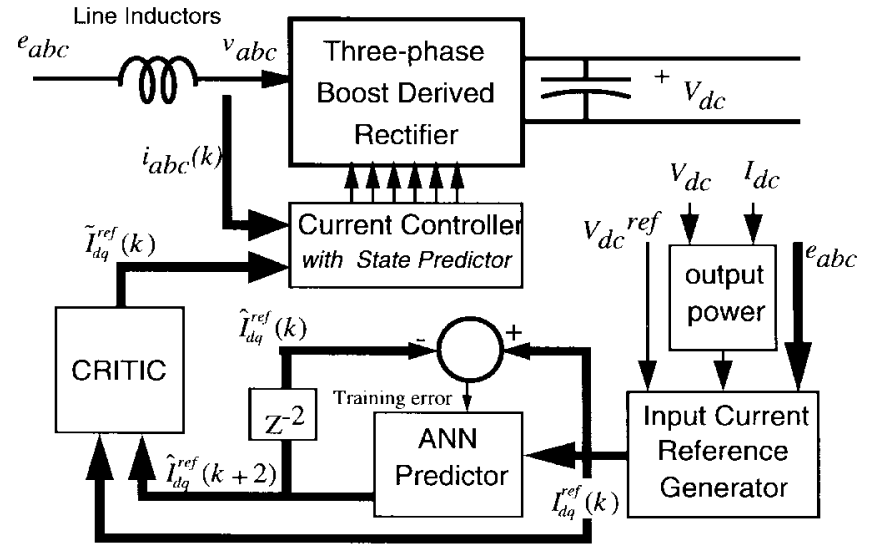

Fig. 1. Rectifier control scheme using a neural-net predictor, illustrating the error to be minimized.

The current regulator typically falls into one of the following two categories: the hysteresis current regulator or one of its improved forms [1], or the predictive deadbeat control [2]. Both techniques have their advantages and disadvantages, and the choice of a particular technique depends upon factors like the switching frequency used, ease of implementation, etc. A number of deadbeat control techniques have been presented previously with increasing degrees of complexity and improved performance. One such technique [2] with good controller performance was subsequently improved [3] by using prediction schemes (see Fig. 1) for the system states and the reference currents which dramatically improved the performance particularly with small dc-bus energy storage. The input-current reference signals $I_{d q}^{\text {ref }}$ in the stationary $d q$ frame of reference are derived from a combination of the dcbus voltage error feedback and the output power feedforward.

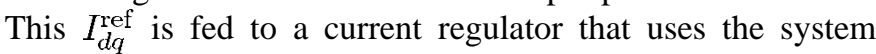
model to compute the required voltage $v_{\mathrm{abc}}$ at the rectifier side of the line inductors: $v_{\text {abc }}$ is realized by space-vector pulse-width modulation (PWM) in the rectifier.

Because of the computation delay, by the time $v_{\text {abc }}$ is calculated, other system variables, i.e., the input currents $i_{\mathrm{abc}}$ and voltages $e_{\text {abc }}$, have changed to new values. In order to fully utilize the sampling time $T_{s}$ for PWM purposes, this computed $v_{\text {abc }}$ is applied at the beginning of the following sampling interval. This results in a control error since the computation of $v_{\text {abc }}$ was based on the actual (old) sampled system variables. However, if one-sampling-interval-aheadpredicted system variables are used in the control computation, it is possible to eliminate or reduce this error. 


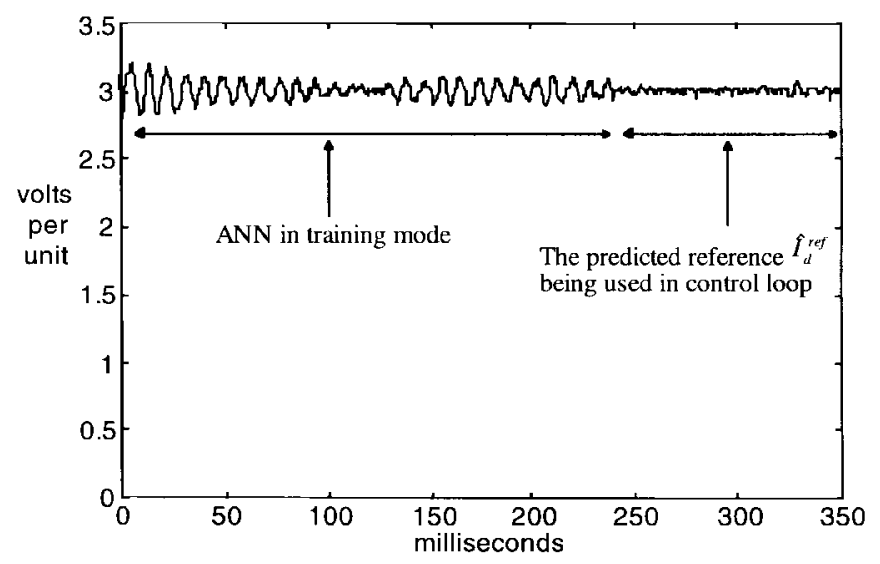

Fig. 2. Rectifier output dc voltage illustrating the improvement in control performance due to state and reference signal prediction.

Another source of error stems from the fact that the $I_{d q}^{\mathrm{ref}}$ computation is based on instantaneous input power demand to supply the outward flow of energy and to compensate for any errors in the dc bus. Because of the computation delay and the fact that the deadbeat control drives the input currents to the reference values in exactly one sampling interval, the input currents actually lag $I_{d q}^{\text {ref }}$ by $4 \pi T s$, where $T_{s}$ is the sampling interval. This lag results in input power being slightly less than the desired power commanded by $I_{d q}^{\text {ref }}$. If the dc-bus energy storage is small compared to the output power, this delay manifests itself as small oscillations on the dc-bus voltage. If the sampling time $T_{s}$ is large or the dc-bus capacitor is too small, these oscillations can cause instability. This error in deadbeat control can be cured by using a $2 T_{s}$ ahead predicted value of $I_{d q}^{\text {ref }}$ in the control computation. Because the $I_{d q}^{\text {ref }}$ calculation requires the output power and the input voltages, which cannot be modeled because of their statistical nature, this prediction of $I_{d q}^{\mathrm{ref}}$ must use statistical or AI methods. An artificial neural-net (ANN)-based predictor was used in [3] along with a state predictor to greatly improve the performance of the rectifier regulator. Fig. 2 shows a typical result where the impact of the prediction schemes on the dc-bus voltage ripple is obvious.

The feedforward ANN in [3] was trained on line using standard backpropagation as opposed to most applications of the feedforward neural nets where training is performed off line using prestored data. In general, each on-line training epoch consists of propagating the ANN input vector forward through the ANN to compute its output, comparing of this output with some reference to compute the training error, and finally modifying the ANN weights in such a way as to reduce the magnitude of this error. Since the ANN input vector changes from one sampling interval to the next, one training epoch has to be completed in one sampling interval. The neural network continuously learns at all times while it is in operation. The typical desired sampling frequency is in the kilohertz range, the corresponding sampling time being in hundreds of microseconds-in [3], the sampling time was $128 \mu \mathrm{s}$. During this sampling time, the processor has to sample, compute the control, and do housekeeping chores-the remaining time is available and can be dedicated to ANN computations-a typical duration being $50 \mu \mathrm{s}$ [3]. Due to the lack of a suitable

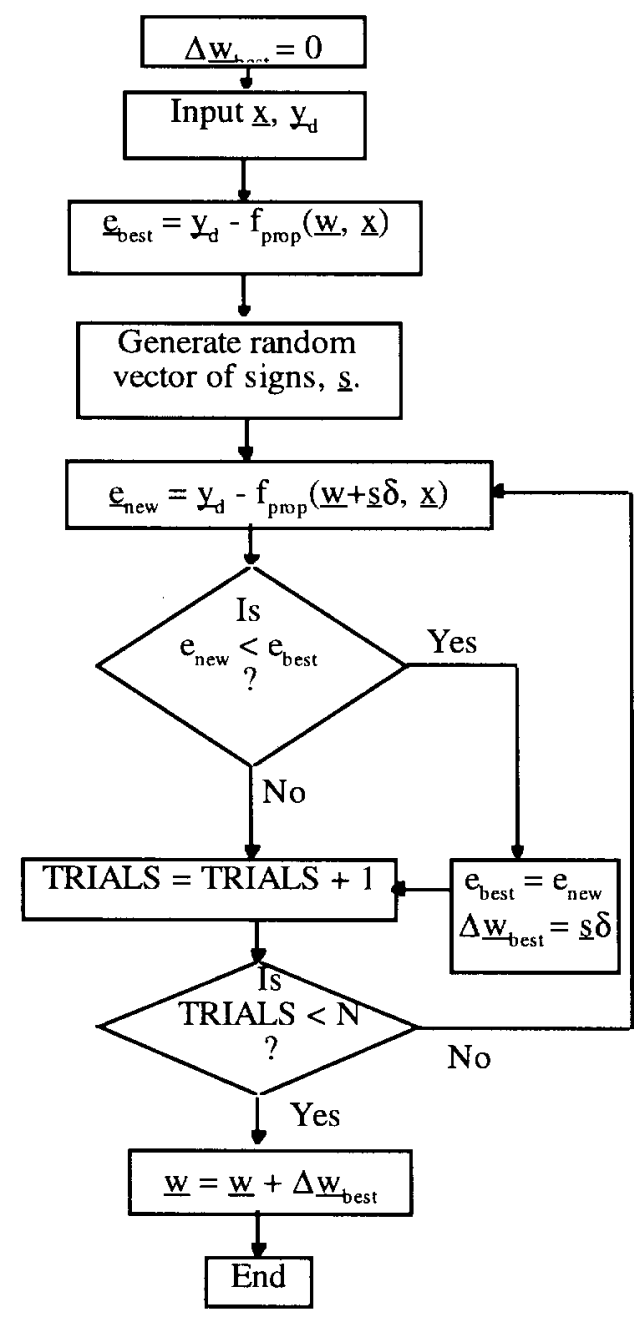

Fig. 3. Flow chart depicting one training epoch of the RWC training algorithm.

ANN ASIC, the ANN of [3] was originally implemented in software on a microprocessor, but for a practical-sized ANN (e.g., 20 inputs, 20 middle-layer nodes, and two outputs), it was not possible to complete all the computations involved in one training epoch during this $50 \mu \mathrm{s}$.

This paper proposes and investigates the use of a new fast on-line training algorithm for feedforward ANN's suitable for hardware implementation and which can meet the timing constraints described above. As opposed to backpropagation, this method is robust and insensitive to the nonidealities of the analog very large-scale integration (VLSI) circuits.

\section{COMPARISON OF AVAILABle Hardware}

Although attempts have been made to implement the feedforward ANN and its backpropagation algorithm in software on single- or multiple-processor arrays [4], the speed achieved is too slow for the real-time application of this rectifier regulator. Higher speeds may be possible using parallel implementations on multiprocessor architectures [5], but the cost of the system becomes prohibitive. Another alternative is the use of ANN ASIC's, but to date, there is no commercial ASIC available that integrates the backpropagation training algorithm along with the forward pass suitable for on-line training at these speeds. More recently, a zero-instruction set 


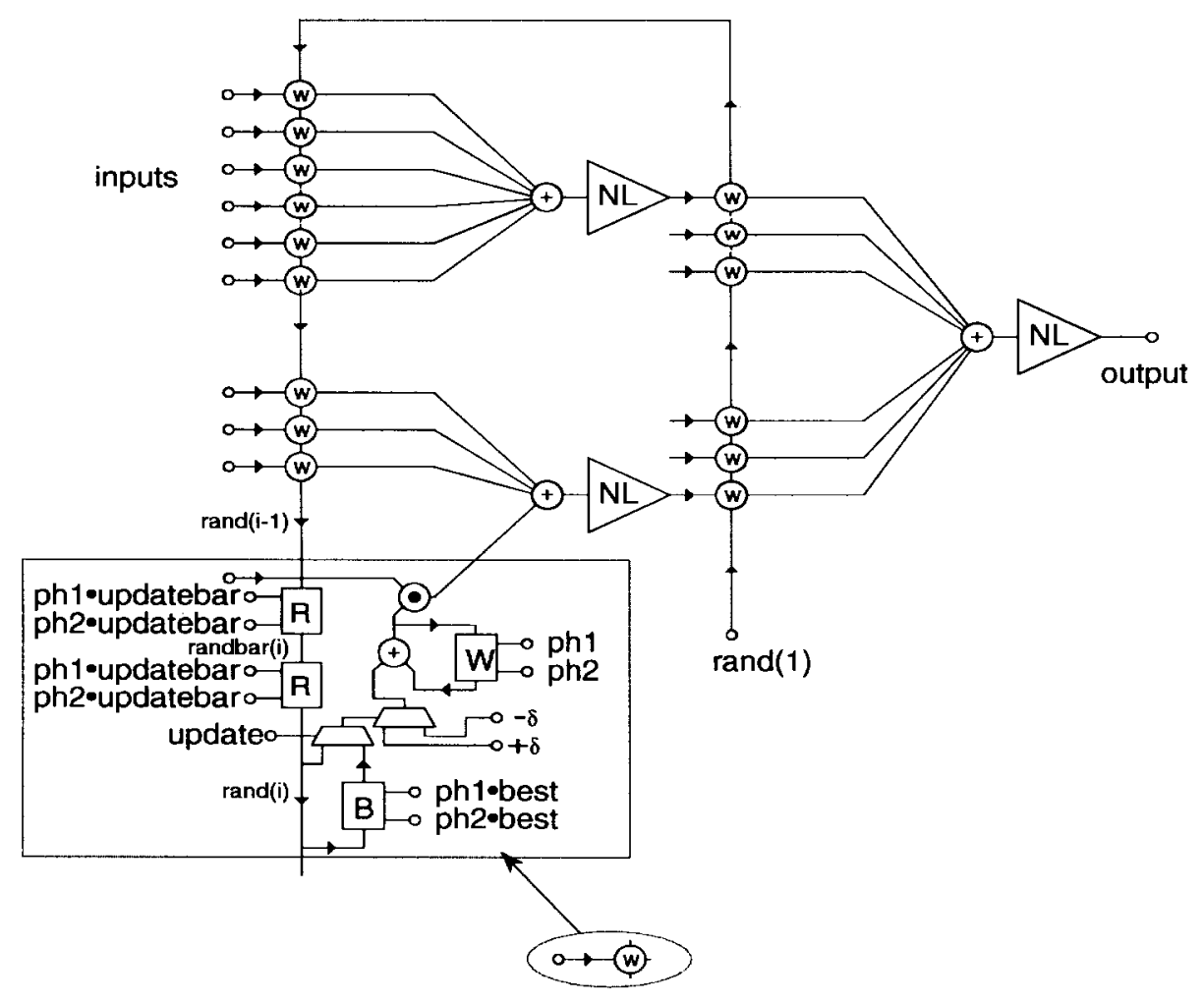

Fig. 4. The RWC learning-hardware schematic.

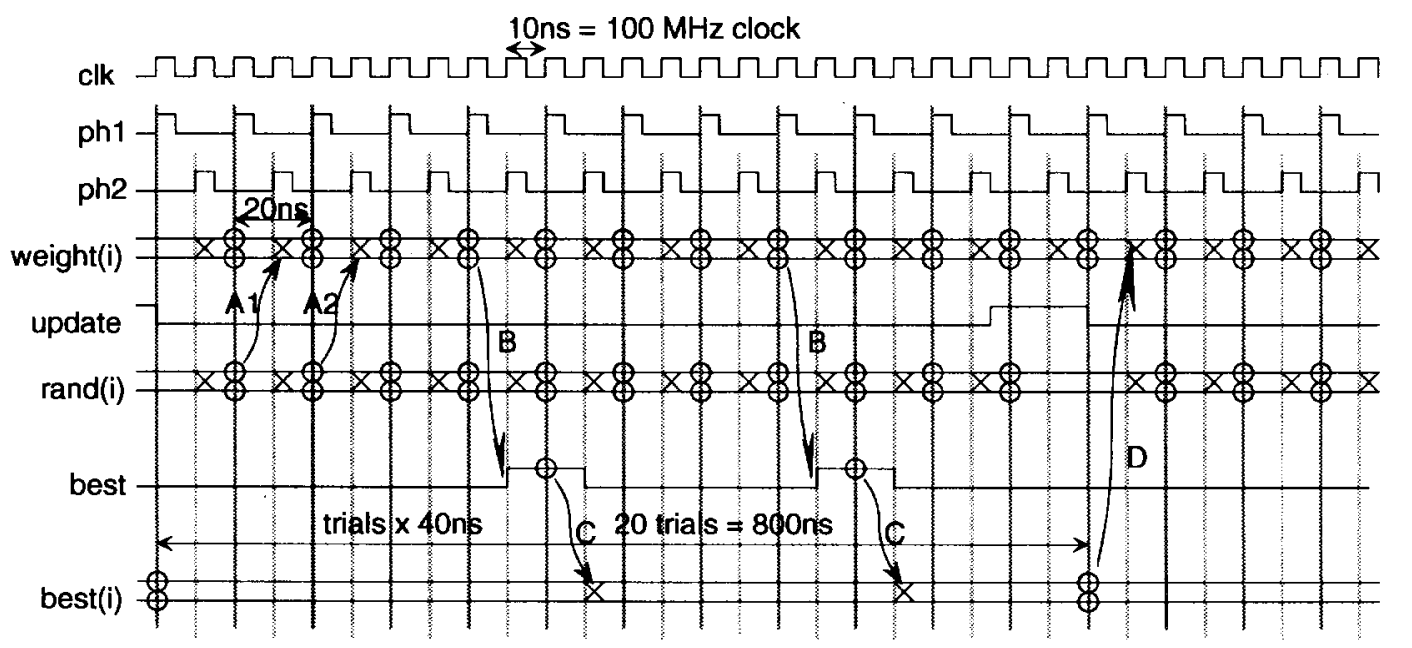

Fig. 5. The RWC learning-hardware timing diagram.

computer chip, ZISC036 was introduced by IBM that uses radial basis functions (RBF's) as the training method [6]; a similar, but more powerful chip using RBF for training has also been developed by Intel and Nestor, Inc. [7]. The radial basis function neural networks are not as powerful as the feedforward ones when compared in terms of extrapolation and generalization capabilities. Another possibility explored by some researchers is to combine a feedforward ANN ASIC like the Intel Electrically Trainable Analog Neural Network (ETANN) with external high-speed processors to implement the backpropagation [8]. The forward pass is carried out in the ETANN, and the weight update computation is done on the external processor. Although this method can achieve considerably higher speeds than a software implementation of the ANN, the achieved speed is still too slow for the rectifier regulator application considered in this paper.

A complete hardware implementation of the feedforward neural net using backpropagation or one of its variants for continuous on-line training has not been accomplished to date. The major obstacle in this regard is the sensitivity of these gradient descent training algorithms to the nonlinearities and offsets present in hardware analog multipliers and adders. The all-digital implementation takes up much larger chip areas than the analog ones and, therefore, a fully parallel digital implementation can be realized only for small networks and with lesser number of bits. Backpropagation is sensitive to the bit resolution and fails to converge if the resolution is inadequate. If instead the computation is carried out serially 
in digital circuits, learning speed must be sacrificed. Analog implementation of weight circuits and multipliers, on the other hand, has the advantage of fast operating speed and small chip areas, therefore allowing larger circuits to be realized. However, the nonidealities of these analog circuits, as mentioned above, render the use of backpropagation or its modified versions unsuitable from a practical standpoint.

All the above problems prompted the search, not for faster hardware to implement continuous on-line training using backpropagation, but instead for an alternate training algorithm which is more robust and less complex and, therefore, would take much less time to execute on VLSI.

\section{The RWC TRAining ALgorithm}

A new ANN training algorithm called random weight change (RWC) has been developed as a variation of a previously proposed method of ANN training based on random search for a minimum on the error surface [9]. As opposed to the deterministic methods of weight training like backpropagation, the RWC algorithm is a statistical or probabilistic method. In very simple terms, during each training cycle or epoch, each of the network weights is perturbed randomly with a fixed magnitude $\pm \delta$, and the ANN output error is computed after the weight change. This error is compared to the value of the previous error before the weight change, and based on this comparison, a decision is taken whether to keep the new weights or not. Keeping the ANN input vector fixed, this process is repeated a number of times called Trials and the final weights are chosen to be the ones that result in the smallest error during one epoch. The flow chart in Fig. 3 explains one training cycle or epoch in more details.

The step size $\delta$ is a training parameter that needs to be determined heuristically for a specific problem. This is similar to the gain coefficient $\beta$ in backpropagation and can be thought of as the radius of a hypersphere in the $n$-dimensional error hypersurface, where $n$ is the total number of network weights. It was observed during experimentation that the value of $\delta$ needs to be small, about two orders of magnitude less than the weight magnitudes. Also, the final convergence is sensitive to $\delta$ which must be determined heuristically for each problem. As is clear from Fig. 3, each epoch contains $N$ trials, therefore, the forward propagation and RWC have to be done $N$ times during each epoch. This appears to be a large amount of computation, but because of the fact that this scheme can be implemented with fully parallel nodes and the random numbers can be generated very efficiently using shift registers, the allhardware implementation can achieve very high speeds. It is important to realize that off line, RWC may take more epochs to converge, but the hardware execution time will make the real training time significantly faster than backpropagation.

\section{RANDOM Weight Change Hardware}

The primary reason for using the RWC algorithm is that the hardware required is significantly easier to fabricate than for backpropagation. To implement backpropagation training in hardware requires high-precision multiplication [9], and this limits the size and/or speed of the hardware that can be fabricated. For applications with hundreds of weights and

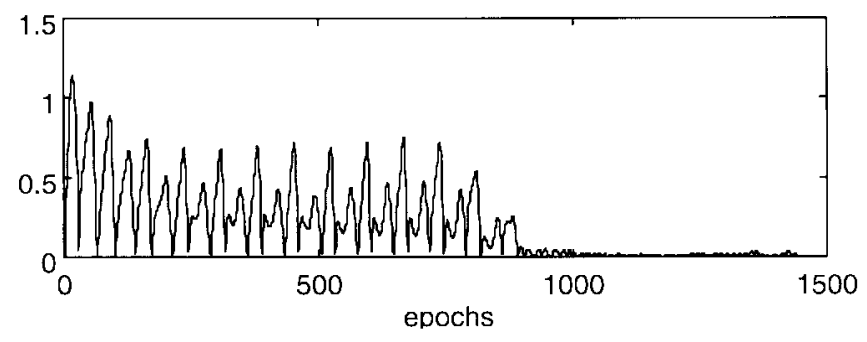

(a)

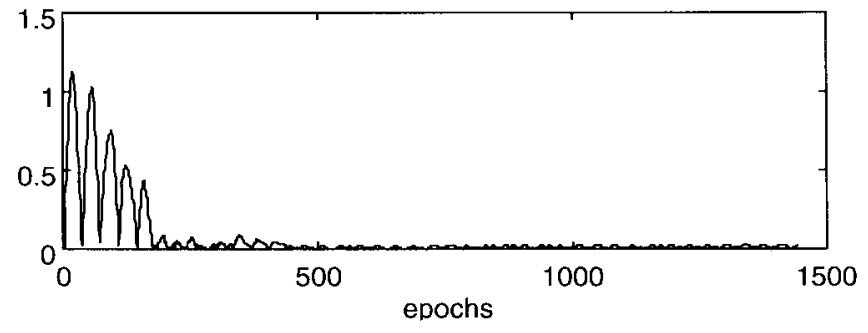

(b)

Fig. 6. Absolute value of output error illustrating training using (a) backpropagation and (b) the RWC algorithm.

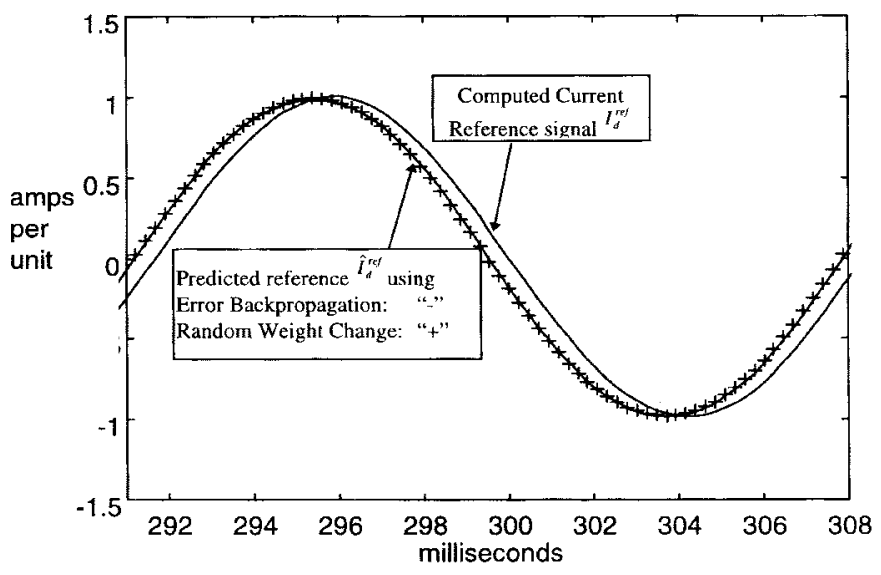

Fig. 7. Computed current reference signal along with the neural-net predicted reference signal using backpropagation and RWC.

weight update times in the microseconds, the RWC algorithm hardware is, therefore, superior in the cost and complexity of the hardware required. This is because compact analog mixedsignal circuitry can be used to perform the weight updates and the forward propagation of the network.

A proposed hardware block schematic for the RWC algorithm learning ANN is presented in Fig. 4. This figure shows the a block diagram of the hardware to implement a conventional feedforward neural network with the RWC training, as shown in Fig. 3. The hardware is controlled by a conventional microcontroller, which generates the clock signals (ph1 and ph2) and a few control signals (update, best, rand, and $\delta$ ). These control signals are changed, at most, every weight update cycle (i.e., epoch) and so do not present a challenge to even low-cost single-chip microcontrollers. The parallel mixed-signal analog-digital hardware carries out all the parallel weight update and forward-propagation operations. It is anticipated that with a $100-\mathrm{MHz}$ digital clock the training epoch, proposed in Fig. 4, would take 800 ns (20 RWC trials). With a less aggressive $10-\mathrm{MHz}$ clock, the epoch would take $8 \mu \mathrm{s}$. 


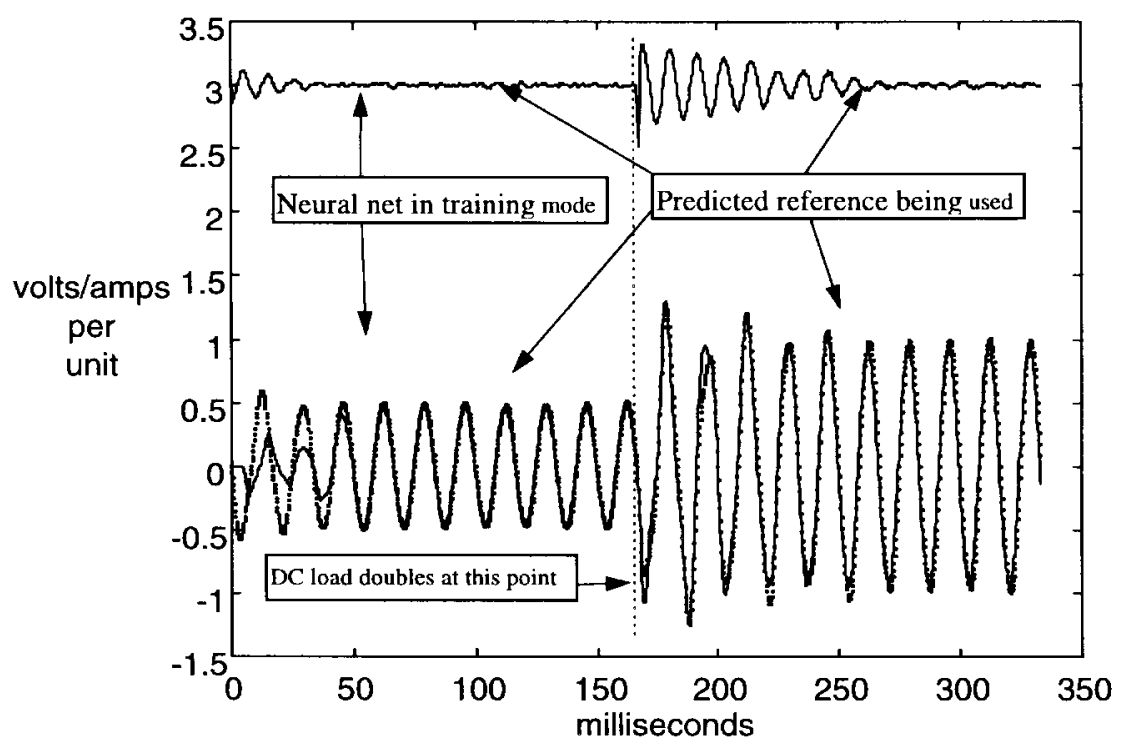

Fig. 8. The dc-bus voltage (upper trace) illustrating the improvement in control performance using the predicted current reference signal (using RWC ANN) with a step change in dc load. Lower traces are the computed current reference signal $I_{d}^{\text {ref } ~ “ . . . " ~ a n d ~ t h e ~ p r e d i c t e d ~ c u r r e n t ~ r e f e r e n c e ~ s i g n a l ~} \hat{I}_{d}^{\text {ref }}$ "-."

Each weight circuit in the ANN contains three registers $(R$, $W$, and $B$ in Fig. 4) that store the RWC's, the current weight, and best weight change for the current epoch. The RWC's are shifted into the register $R$ and used to update the weight register $W$. Then, the opposite weight change is shifted into $R$ and used to return $W$ to the original value. The timing of this process is shown in Fig. 5. The step A1 represents the trial of the current weight change, while A2 represents the return to the original value of the weights.

The external signal "best" is computed externally from the hardware and indicates that the current weight change is the best so far among the completed trials in the current epoch. This signal causes the current weight change to be saved in the $B$ (best) register. When one epoch is complete, the update signal is raised and the best weight change is permanently saved in the weight register $(W)$. In step $B$, the external processor computes that the current weight change is the best for the epoch. In step $C$, the value of the register $B$ [best( i)] is updated. In step $D$, the best overall weight change is made permanent.

\section{RESULTS}

In order to assess the suitability of the RWC algorithm for the rectifier regulator prediction problem, a computer simulation was performed. The ANN used in the simulation consists of 20 inputs, 20 middle-layer neurons, and two outputs. The middle-layer outputs pass through sigmoidal nonlinearities while the final outputs are linear. The complete system of Fig. 1 consisting of the three-phase rectifier along with the deadbeat controller and the ANN predictor was simulated. The neural network, which serves as a time-series predictor of the input-current reference, uses the previous ten values of $I_{d q}^{\text {ref }}$ as ANN inputs for one epoch. During each epoch, the process shown in Fig. 3 is repeated for $N=20$ times, keeping constant the inputs for that epoch.

For comparison purposes, the simulation was repeated with an identical rectifier system and the same ANN, using backpropagation for training. Fig. 6 compares the output errors produced by the two (RWC and backpropagation) training methods as a function of the number of epochs. Clearly, the RWC method converges faster than backpropagation.

Fig. 7 shows the computed current reference $I_{d}^{\text {ref }}$ and the predicted current reference signal $\hat{I}_{d}^{\text {ref }}$ using the two training algorithms. Note in Fig. 7 that both the backpropagation method and RWC method predict essentially the same current reference and that the predicted current reference leads the computed reference as expected. The good agreement between the results of Figs. 6 and 7 prove that the new fast on-line random training method gives results which are as good as those obtained using backpropagation. Fig. 8 illustrates the effect of the RWC-based predictor on the rectifier dc-output voltage in the presence of a load change, which causes the ANN to retrain. The ANN size and training parameter $\delta$ are chosen by trial and error. There is a minimum required number of nodes determined by the problem complexity. A network with smaller size cannot retain the information necessary for determining the correct response. This number is determined through experiments. Similarly, the convergence speed and the final resulting error are functions of the training parameter $\delta$ and the number of trials. These parameters were also determined through experiments.

Although this paper does not include results for varying network size and the effect of $\delta$, a similar study was performed on an induction motor control problem. Results have been presented in [10] showing that an ANN with 12 nodes in the inner layer can be trained to identify and control the motor currents almost as well as one with 30 nodes. Moreover, the value of the training step size $\delta$ influences the system behavior in the same way as the proportion gain of a traditional feedback controller.

\section{CONCLUSION}

A new fast on-line training algorithm for feedforward artificial neural networks with a potential for hardware realizability has been proposed for use in a rectifier control scheme. The algorithm was demonstrated to produce results comparable to 
backpropagation when applied to a three-phase rectifier control problem. The algorithm is based on the method of random search, is computationally very simple, and therefore suitable for VLSI implementation.

\section{REFERENCES}

[1] L. Malesani, L. Rossetto, P. Tenti, P. Tomasin, and A. Zuccato, "Performance optimization of quasidirect converters," in PCC Yokohama, 1993, pp. 99-104.

[2] T. G. Habetler, "A space vector based rectifier regulator for ac/dc/ac converters," IEEE Trans. Power Electron., vol. 8, no. 1, pp. 30-36, 1993.

[3] F. Kamran and T. G. Habetler, "An improved dead-beat rectifier regulator using a neural net predictor," in IEEE Power Electronics Specialists Conf., Taiwan, 1994, pp. 1431-1436.

[4] B. Burton, R. G. Harley, and G. Diana. J. L. Rodgerson, "Implementation of a neural network to adaptively identify and control VSI fed induction motor stator currents," in Conf. Rec. IEEE IAS Annu. Meet., Denver, CO, Oct. 1994, pp. 1733-1740.

[5] V. Kumar, S. Shekhar, and N. B. Amin, "A scalable parallel formulation of the back propagation algorithm for hypercubes and related architectures," IEEE Trans. Parallel Distrib. Syst., vol. 5, no. 10, pp. 1073-1090, 1994

[6] ̊.. Eide, Th. Lindblad, C. S. Lindsey, M. Minerskjöld, G. Sekhniaidze, and G. Székely, "An implementation of the zero instruction set computer (ZISC036) on a PC/ISA-bus card," WNN/FNN, Washington DC, Dec. 1994.

[7] C. Park, K. Buckmann, J. Diamond, U. Santoni, S.-C. The, M. Holler, M. Glier, C. L. Scofield, and L. Nunez, "A radial basis function neural network with on-chip learning," in Proc. ICJNN, Japan, Oct. 1993, pp. 3035-3038.

[8] G. Cancelo and S. Hansen, "Analog neural network development system with fast on line training capabilities," in IEEE IECON, Italy, Sept. 1994, pp. 1396-1400.

[9] K. Hirotsu and M. Brooke, "An analog neural network chip with random weight change learning algorithm," in Proc. ICJNN, Japan, Oct. 1993, pp. 3031-3034.

[10] B. Burton, F. Kamran, R. G. Harley, T. G. Habetler, M. Brooke, and R. Poddar, "Identification and control of induction motor stator currents using fast on-line random training of a neural network," to be published.

Farrukh Kamran (S'94) received the B.Sc. degree in electrical engineering from the University of Engineering and Technology, Lahore, Pakistan, in January 1988 and the M.S.E.E. and Ph.D. degrees from the Georgia Institute of Technology, Atlanta, in 1992 and 1995, respectively.

After graduating with the B.Sc. degree, he served as Design Engineer with EGS (pvt) Ltd., Pakistan, from 1988 to 1989. He joined HiPo Electronics (pvt) Ltd., Pakistan, in 1989 as a Senior Design Engineer in power electronics, where he served until 1991. He is currently an Assistant Professor in the Faculty of Electronics, Ghulam Ishaq Khan Institute of Engineering Sciences and Technology, Pakistan. He is engaged in research on controlled rectifiers, active power filters, line conditioners, and UPS's.

Dr. Kamran received a scholarship for graduate studies by the Government of Pakistan. He is a Member of the Pakistan Engineering Council.

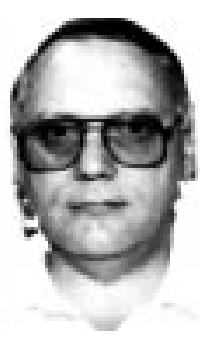

Ronald G. Harley (M'77-SM'86-F'92) received the M.Sc.Eng. degree from the University of Pretoria, South Africa, in 1965 and the Ph.D. degree from Imperial College, London, U.K., in 1969.

In 1971, he was appointed Professor of Electrical Machines and Control at the University of Natal, Durban, South Africa. He was a Visiting Professor at Iowa State University, Ames, in 1977, Clemson University, Clemson, SC, in 1987, and the Georgia Institute of Technology, Atlanta, in 1994. His areas of research include power system dynamics, electrical machines, power electronics, and control of ac variable-speed drives.

Dr. Harley is a Member of PES and IAS. He is also a Fellow of the IEE and SAIEE, South Africa.

Bruce Burton (S'94) was born in Johannesburg, South Africa, in 1971. $\mathrm{He}$ received the B.Sc.Eng. degree in electronic engineering in 1992 and the M.Sc.Eng. degree in electrical engineering in 1995, both from the University of Natal, South Africa. He is presently working toward the Ph.D. degree at the same university.

His research interests include artificial intelligence, control systems, electrical drives, and digital controller hardware.

Thomas G. Habetler (S'82-M'83-SM'92) received the B.S.E.E. degree in 1981 and the M.S. degree in 1984, both in electrical engineering, from Marquette University, Milwaukee, WI, and the Ph.D. degree from the University of Wisconsin, Madison, in 1989.

From 1983 to 1985, he was with the Electro-Motive Division of General Motors as a Project Engineer. While there, he was involved in the design of switching power supplies and voltage regulators for locomotive applications. $\mathrm{He}$ is currently an Associate Professor of Electrical Engineering at the Georgia Institute of Technology, Atlanta. His research interests are in switching converter technology and electric machine protection and drives.

Dr. Habetler was Corecipient of the 1989 First-Prize Paper Award, 1991 Second-Prize Paper Award of the Industrial Drives Committee, and 1994 Second-Prize Paper Award of the Electric Machines Committee of the IEEE Industry Applications Society. He is an Associate Editor of the IEEE TRANSACtions on POWER Electronics. He also serves as Educational Activities Chair of the IEEE Power Electronics Society.

Martin A. Brooke (M'87) received the B.E. (Elect.) degree (1st-Class Hons.) from Auckland University, New Zealand, in 1981 and the M.S. and Ph.D. degrees in electrical engineering from the University of Southern California, Los Angeles, in 1984, and 1988, respectively.

$\mathrm{He}$ is currently Associate Professor of Electrical Engineering at the Georgia Institute of Technology, Atlanta. He has four U.S. patents. He publishes in a variety of technical journals, and articles on his work have appeared in several trade publications.

Dr. Brooke won a National Science Foundation Research Initiation Award in 1990 and the 1992 IEEE Midwest Symposium on Circuits and Systems Myril B. Reed Best Paper Award. He is a Member of the INNS. 